\title{
INTERPOLATING VARIETIES FOR WEIGHTED SPACES OF ENTIRE FUNCTIONS IN $\mathrm{C}^{n}$
}

\author{
Carlos A. Berenstein and Bao Qin Li
}

\begin{abstract}
We prove in this paper that a given discrete variety $V$ in $\mathbf{C}^{n}$ is an interpolating variety for a weight $p$ if and only if $V$ is a subset of the variety $\left\{\xi \in \mathbf{C}^{n}: f_{1}(\xi)=f_{2}(\xi)=\cdots=f_{n}(\xi)=0\right\}$ of $m$ functions $f_{1}, \cdots, f_{m}$ in the weighted space the sum of whose directional derivatives in absolute value is not less than $\epsilon \exp (-C p(\zeta)), \quad \zeta \in$ $V$ for some constants $\epsilon, C>0$. The necessary and sufficient conditions will be also given in terms of the Jacobian matrix of $f, \cdots, f_{m}$. As a corollary, we solve an open problem posed by Berenstein and Taylor about interpolation for discrete varieties.
\end{abstract}

1. Introduction. In this note, we shall study the problem of finding necessary and sufficient conditions for a given discrete subset of $\mathbf{C}^{n}$ to be interpolating for spaces of entire functions in several complex variables satisfying growth conditions.

Let $V=\left\{\zeta_{k}\right\}$ be a discrete subset of $\mathbf{C}^{n}$ and $p(\xi), \xi=\left(\xi_{1}, \cdots, \xi_{n}\right) \in$ $\mathbf{C}^{n}$, a plurisubharmonic weight function on $\mathbf{C}^{n}$. We consider the following interpolation problem: under what conditions is it true that for any sequence $\left\{a_{k}\right\}$ of complex numbers satisfying the growth condition

$$
\left|a_{k}\right| \leq A \exp \left(B p\left(\zeta_{k}\right)\right), \quad k \in \mathbf{N}:=\{1,2, \cdots\}
$$

for some constants $A, B>0$, there exists an entire function $f$ on $\mathbf{C}^{n}$ such that $f\left(\zeta_{k}\right)=a_{k}$ and $f$ satisfies the same kind of estimate for $\xi \in \mathbf{C}^{n}$, namely, $f \in A_{p}\left(\mathbf{C}^{n}\right)$ or equivalently

$$
|f(\xi)| \leq A^{\prime} \exp \left(B^{\prime} p(\xi)\right)
$$

This research is supported in part by NSF grants DMS 92-25043 and CDR 88-03012. 
for some constants $A^{\prime}, B^{\prime}>0$. We will then say that $V$ is an interpolating variety (for the weight $p$ or the space $A_{p}\left(\mathbf{C}^{n}\right)$ ).

In the case when $V$ is a complete intersection and defined by so-called slowly decreasing functions, some interpolation results have been known in [BT2], where a vector function $F=\left(F_{1}, F_{2}, \cdots, F_{n}\right), F_{i} \in A_{p}\left(\mathbf{C}^{n}\right)$, is called slowly decreasing if and only if there exist $\epsilon, C, C_{1}, C_{2}>0$ such that

(i) the connected components of the set $S(F ; \epsilon, C)$ are bounded, where $S(F ; \epsilon, C)=\left\{\xi \in \mathbf{C}^{n}:|F(\xi)|=\left(\sum_{j=1}^{n}\left|F_{j}(\xi)\right|^{2}\right)^{1 / 2}<\right.$ $\epsilon \exp (-C p(\xi))\} ;$ and

(ii) if $\Omega$ is a component of $S(F ; \epsilon, C)$, then $p(\xi) \leq C_{1} p(w)+C_{2}$, for all $\xi, w \in \Omega$.

Note that the definition depends on the weight function $p . \quad F$ may be slowly decreasing for one weight and not for another. We refer to [BT2] for a class of examples of slowly decreasing functions. With this notion, the interpolation theorem in [BT2] about discrete varieties can be stated as follows:

Let $F=\left(F_{1}, F_{2}, \cdots, F_{n}\right), F_{i} \in A_{p}\left(\mathbf{C}^{n}\right)$, be slowly decreasing. Assume further that the points $\left\{\zeta_{k}\right\}$ of $F(\xi)=0$ are simple; that is,

$$
\operatorname{det} J_{F}\left(\zeta_{k}\right) \neq 0 \quad\left(J_{F}(\xi)=\text { Jacobian matrix of } F\right) .
$$

Then $V=\left\{\zeta_{k}\right\}$ is interpolating for $A_{p}\left(\mathbf{C}^{n}\right)$ if and only if there exist constants $\epsilon>0, C>0$ such that

$$
\left|\operatorname{det} J_{F}\left(\zeta_{k}\right)\right| \geq \epsilon \exp \left(-C p\left(\zeta_{k}\right)\right), \quad k \in \mathbf{N} .
$$

For $n=1, p(z)=p(|z|)$, this result is due to A. F. Leont'ev [L]. For $n=1, p(z)=|\operatorname{Im} z|+\log (1+|z|)$, this result is due to Ehrenpreis and Malliavin [EM]. For $n=1$ and general weights $p$, this result is given in [BT1].

Note that the above result only applies to the case of discrete varieties $V=\left\{\zeta_{k}\right\}$ which are exactly complete intersections of $n$ slowly decreasing functions $F_{1}, F_{2}, \cdots, F_{n}$, i.e.,

$$
V=Z\left(F_{1}, F_{2}, \cdots, F_{n}\right):=\left\{\xi \in \mathbf{C}^{n}: F_{1}(\xi)=F_{2}(\xi)=\cdots=F_{n}(\xi)\right\}
$$

with $F=\left(F_{1}, F_{2}, \cdots, F_{n}\right)$ slowly decreasing. It was pointed out in [BT2, p. 210] that the case when the variety is not such a complete intersection seems to be quite different. Therefore it is a natural goal to try to find conditions without the above hypotheses on the varieties, in particular, to find conditions necessary and sufficient for interpolation 
which apply to any given discrete variety. We shall solve this problem in the present note. It turns out, roughly speaking, that a discrete variety $V$ is interpolating for $p$ if and only if $V$ is a subset of the analytic variety $Z\left(f_{1}, \cdots, f_{m}\right)$ for $m(\geq n)$ functions $f_{1}, \cdots, f_{m} \in A_{p}$ the sum of whose directional derivatives in absolute value is not "too small" (see Theorem 2.5). As a corollary, we solve an open problem posed by Berenstein and Taylor in [BT3, p. 10] about interpolation for discrete varieties (see Remark 2.8).

2. Definitions and results. First of all, let us fix the notations which we shall use throughout this paper.

A plurisubharmonic function $p: \mathbf{C}^{n} \rightarrow[0, \infty)$ is called a weight function if it satisfies the following conditions (c.f. [BT2]):

$$
\log \left(1+|\xi|^{2}\right)=O(p(\xi))
$$

and there exist constants $C_{1}$ and $C_{2}$ such that $|\xi-w| \leq 1$ implies

$$
p(\xi) \leq C_{1} p(w)+C_{2}
$$

Definition 2.1. Let $A\left(\mathbf{C}^{n}\right)$ be the ring of all entire functions on $\mathbf{C}^{n}$.Then

$$
\begin{aligned}
A_{p} & =A_{p}\left(\mathbf{C}^{n}\right)= \\
& =\left\{f \in A\left(\mathbf{C}^{n}\right):|f(\xi)| \leq A \exp (B p(\xi)) \text { for some } A, B>0\right\}
\end{aligned}
$$

It is not the specific conditions on $p$ which are important, but rather their consequences for the ring $A_{p}$. It follows from (2.1) that $A_{p}\left(\mathbf{C}^{n}\right)$ contains the polynomials and, from (2.2), that $f \in A_{p}\left(\mathbf{C}^{n}\right)$ implies $\frac{\partial f}{\partial \xi_{k}} \in A_{p}\left(\mathbf{C}^{n}\right)$ (see e.g. [Ho]). One can replace (2.2) by the following Hörmander's condition ([Ho]): there exist four positive constants $c_{1}, \cdots, c_{4}$ such that $|\xi-w| \leq \exp \left(-c_{1} p(w)-c_{2}\right)$ implies that $p(\xi) \leq c_{3} p(w)+c_{4}$. We use (2.2) only for the sake of convenience.

Remark 2.2. The two basic examples of such weight functions are $p(\xi)=|\xi|^{\rho}(\rho>0)$ and $p(\xi)=|\operatorname{Im} \xi|+\log \left(1+|\xi|^{2}\right)$ corresponding to the space $A_{p}$ of all entire functions of order $\leq \rho$ and finite type and the space $\widehat{\mathcal{E}}^{\prime}\left(\mathbf{R}^{n}\right)$ of Fourier transforms of distributions with compact support in $\mathbf{R}^{n}$ (see e.g. [E]).

Definition 2.3. Let $V=\left\{\zeta_{k}\right\}$ be a discrete variety on $\mathbf{C}^{n}$, i.e., a discrete sequence in $\mathbf{C}^{n}$ with $\left|\zeta_{k}\right| \uparrow \infty$ as $k \rightarrow \infty$. Then

$$
A_{p}(V)=\left\{a=\left\{a_{k}\right\}_{k \in \mathbf{N}}: \exists A, B>0,\left|a_{k}\right| \leq A \exp \left(B p\left(\zeta_{k}\right)\right), \forall k \in \mathbf{N}\right\} .
$$


With the above definitions, the interpolation problem is simply to determine when the following restriction map $\rho: A_{p} \rightarrow A_{p}(V)$ defined by $\rho(f)=\left\{f\left(\zeta_{k}\right)\right\}$, is onto from $A_{p}$ to $A_{p}(V)$.

Definition 2.4. A discrete variety $V=\left\{\zeta_{k}\right\}$ is an interpolating variety for $A_{p}=A_{p}\left(\mathbf{C}^{n}\right)$ if the restriction map $\rho$ is onto from $A_{p}$ to $A_{p}(V)$.

Now we can state our main theorem and its corollaries, whose proofs will be given in the next section.

Theorem 2.5. Let $V=\left\{\zeta_{k}\right\}$ be a discrete variety on $\mathbf{C}^{n}$ and $m(\geq n)$ be an integer. Then $V$ is an interpolating variety for $A_{p}\left(\mathbf{C}^{n}\right)$ if and only if there exist $m$ functions $f_{1}, f_{2}, \cdots, f_{m} \in A_{p}\left(\mathbf{C}^{n}\right)$ such that

$$
V \subset Z\left(f_{1}, f_{2}, \cdots, f_{m}\right)
$$

and for some $\epsilon, C>0$

$$
\sum_{j=1}^{m}\left|D_{u} f_{j}\left(\zeta_{k}\right)\right| \geq \epsilon \exp \left(-C p\left(\zeta_{k}\right)\right), \forall k \in \mathbf{N}, u \in \mathbf{S}^{2 n-1},
$$

where $D_{u} f(\xi):=\frac{\partial f}{\partial \xi_{1}} u_{1}+\cdots+\frac{\partial f}{\partial \xi_{n}} u_{n}$ is the directional derivative of $f$ along the direction $u \in \mathbf{S}^{2 n-1}$. Here

$$
\mathbf{S}^{2 n-1}:=\left\{\xi=\left(\xi_{1}, \cdots, \xi_{n}\right):|\xi|:=\left(\left|\xi_{1}\right|^{2}+\cdots+\left|\xi_{n}\right|^{2}\right)^{\frac{1}{2}}=1\right\} .
$$

Corollary 2.6. Let $V=\left\{\zeta_{k}\right\}$ be a discrete subset of $\mathbf{C}^{n}$ and $m(\geq n)$ be an integer. Then $V$ is an interpolating variety for $A_{p}\left(\mathbf{C}^{n}\right)$ if and only if there exist $m$ functions $f_{1}, f_{2}, \cdots, f_{m} \in A_{p}$ such that

$$
V \subset Z\left(f_{1}, f_{2}, \cdots, f_{m}\right)
$$

and for each $k \in \mathbf{N}$, there exists an $n \times n$ minor $\mathcal{J}$ of the Jacobian matrix of $f_{1}, \cdots, f_{m}$ such that

$$
\left|\operatorname{det} \mathcal{J}\left(\zeta_{k}\right)\right| \geq \epsilon \exp \left(-C p\left(\zeta_{k}\right)\right)
$$

where $\epsilon, C$ are two positive constants independent of $k$.

Corollary 2.7. Let $V=\left\{\zeta_{k}\right\}$ be a discrete subset of $\mathbf{C}^{n}$. Then $V$ is an interpolating variety for $A_{p}\left(\mathbf{C}^{n}\right)$ if and only if there exist $n$ functions $f_{1}, f_{2}, \cdots, f_{n} \in A_{p}$ such that

$$
V \subset Z\left(f_{1}, f_{2}, \cdots, f_{n}\right)
$$


and for some $\epsilon, C>0$

$$
\left|\operatorname{det} J_{f_{1}, \cdots, f_{n}}\left(\zeta_{k}\right)\right| \geq \epsilon \exp \left(-C p\left(\zeta_{k}\right)\right), \quad k \in \mathbf{N},
$$

where $J_{f_{1}, \cdots, f_{n}}$ is the Jacobian matrix of $f_{1}, \cdots, f_{n}$.

Let us mention here that the sufficiency of Corollary 2.6 or 2.7 can not follow from [BT3] where the variety was again restricted to be the complete intersection of some functions in $A_{p}$. In the case $n=1$, a stronger version of Corollary 2.7 which allows arbitrary multiplicities can be given. We refer the reader to our papers $[\mathbf{B L} \mathbf{1}],[\mathbf{B L 2}]$ and $[\mathbf{B L V}]$ for related results in this direction.

Remark 2.8. Observe that when the conditions (2.3) and (2.4) are satisfied for a weight $p$, they are automatically satisfied for any weight $q \geq p$. This gives an affirmative answer to an open problem in [BT3, p. 10] for discrete varieties: Let $V$ be an interpolating variety for $A_{p}\left(\mathbf{C}^{n}\right)$ and $q$ another weight satisfying $q \geq p$. Is $V$ interpolating for $A_{q}\left(\mathbf{C}^{n}\right)$ ?

We conclude this section by providing an interpolation example using Theorem 2.5.

Example 2.9. Let $p_{j}: \mathbf{C} \rightarrow[0, \infty)$ be weights in $\mathbf{C}(1 \leq j \leq n)$ and $V_{j}=\left\{z_{k, j}\right\}_{k=1}^{\infty}$ be an interpolating variety for $A_{p_{j}}(\mathbf{C})$. Then we claim that

$$
V:=\left\{\zeta_{k}=\left(\zeta_{k, 1}, \cdots, \zeta_{k, n}\right): \zeta_{k, j} \in V_{j}\right\}_{k=1}^{\infty}
$$

is an interpolating variety for $A_{p}\left(\mathbf{C}^{n}\right)$, where $p(\xi)=p_{1}\left(\xi_{1}\right)+\cdots+p_{n}\left(\xi_{n}\right)$ for $\xi=\left(\xi_{1}, \cdots, \xi_{n}\right)$.

In fact, since $V_{j}$ is an interpolating variety for $A_{p_{j}}$, we know that by Corollary 2.7, there exists an entire function $f_{j}(z)$ in $A_{p_{j}}(\mathbf{C})$ such that $V_{j} \subset Z\left(f_{j}\right)$ and for some $\epsilon_{j}, c_{j}>0$,

$$
f_{j}^{\prime}\left(\zeta_{k, j}\right) \geq \epsilon_{j} \exp \left(-c_{j} p_{j}\left(\zeta_{k, j}\right)\right) .
$$

Let

$$
F_{j}(\xi)=f_{j}\left(\xi_{j}\right) \quad(1 \leq j \leq n) .
$$

Then clearly, $F_{j} \in A_{p}\left(\mathbf{C}^{n}\right)$ and $V \subset Z\left(F_{1}, \cdots, F_{n}\right)$. Moreover,

$$
\left|\operatorname{det} J_{F}\left(\zeta_{k}\right)\right| \geq \epsilon \exp \left(-c p\left(\zeta_{k}\right)\right) \text {, }
$$

with $c=\max c_{j}, \epsilon=\epsilon_{1} \cdots \epsilon_{n}$. Now we conclude by Corollary 2.7 that $V$ is an interpolating variety for $A_{p}\left(\mathbf{C}^{n}\right)$. Furthermore, $V_{1} \times \cdots \times V_{n}$ is also an interpolation variety. As a concrete example, we see that the lattice

$$
\begin{aligned}
V & =\mathcal{Z}^{2} \times \cdots \times \mathcal{Z}^{2}= \\
& =\left\{\left(\zeta_{1}, \cdots, \zeta_{n}\right) \in \mathbf{C}^{n}: \zeta_{j} \in \mathcal{Z}^{2}, 1 \leq j \leq n\right\},
\end{aligned}
$$


where $\mathcal{Z}^{2}=\{m+i n: m, n \in \mathbf{Z}\}$, is an interpolating variety for $A_{p}\left(\mathbf{C}^{n}\right)$ with $p(\xi)=|\xi|^{2}=\left|\xi_{1}\right|^{2}+\cdots+\left|\xi_{n}\right|^{2}$ for $\xi=\left(\xi_{1}, \cdots, \xi_{n}\right) \in \mathbf{C}^{n}$, since it is known that $\mathcal{Z}^{2}$ is an interpolating variety for $A_{p}(\mathbf{C})$ with $p(z)=|z|^{2}$ for $z \in \mathbf{C}$.

3. Proofs of Theorem 2.5 and its corollaries. Let us first prove the following lemmas.

Lemma 3.1. Let $\left\{\zeta_{k}\right\}$ be a discrete subset of $\mathbf{C}^{n}$ and $\delta_{k}=$ $\inf _{j \neq k}\left\{\left|\zeta_{j}-\zeta_{k}\right|\right\}$. If for some constants $\epsilon, B>0$

$$
\delta_{k} \geq \epsilon \exp \left(-B p\left(\zeta_{k}\right)\right), k \in \mathbf{C}
$$

then there exists a $M>0$ such that

$$
\sum_{k=1}^{\infty} \exp \left(-M p\left(\zeta_{k}\right)\right)<\infty .
$$

Proof: Denote

$$
\eta_{k}=\min \left\{1, \delta_{k}\right\}
$$

and

$$
\mathcal{B}_{k}=\mathcal{B}\left(\zeta_{k}, \eta_{k}\right):=\left\{\xi \in \mathbf{C}^{n}:\left|\xi-\zeta_{k}\right| \leq \eta_{k}\right\} .
$$

Let $d V(\xi)$ be the Euclidean volume element in $\mathbf{C}^{n}$ and $\left|\mathcal{B}_{k}\right|=\int_{\mathcal{B}_{k}} d V(\xi)$. Then (3.1) implies that

$$
\left|\mathcal{B}_{k}\right| \geq \epsilon_{1} \exp \left(-B_{1} p\left(\zeta_{k}\right)\right)
$$

for some positive numbers $\epsilon_{1}, B_{1}$. We then conclude that for large $M$,

$$
\begin{aligned}
\sum_{k=1}^{\infty} \exp \left(-M p\left(\zeta_{k}\right)\right) & =\sum_{k=1}^{\infty} \frac{1}{\left|\mathcal{B}_{k}\right|} \int_{\mathcal{B}_{k}} \exp \left(-M p\left(\zeta_{k}\right)\right) d V(\xi) \leq \\
& \leq \frac{1}{\epsilon_{1}} \sum_{k=1}^{\infty} \int_{\mathcal{B}_{k}} \exp \left(\left(B_{1}-M\right) p\left(\zeta_{k}\right)\right) d V(\xi) \leq \\
& \leq \frac{C_{2}\left(B_{1}-M\right)}{\epsilon_{1}} \sum_{k=1}^{\infty} \int_{\mathcal{B}_{k}} \exp \left(C_{1}\left(B_{1}-M\right) p(\zeta)\right) d V(\xi) \leq \\
& \leq \frac{C_{2}\left(B_{1}-M\right)}{\epsilon_{1}} \int_{\mathbf{C}}^{n} \exp \left(C_{1}\left(B_{1}-M\right) p(\zeta)\right) d V(\xi)<\infty
\end{aligned}
$$

by virtue of the property (2.2) and (2.1) of the weight $p$. 
Lemma 3.2. Let $f_{1}, \cdots, f_{m}$ be $m(\geq n)$ entire functions in $\mathbf{C}^{n}$. Then

$$
\sum_{j=1}^{m}\left|D_{u} f_{j}\left(\zeta_{k}\right)\right| \geq \epsilon \exp \left(-C p\left(\zeta_{k}\right)\right), \forall k \in \mathbf{N}, u \in \mathbf{S}^{2 n-1}
$$

for some constants $\epsilon, C>0$ if and only if for each $k \in \mathbf{N}$, there exists a $n \times n$ minor $\mathcal{K}$ of the Jacobian matrix $J_{f_{1} \cdots f_{m}}$ of $f_{1}, \cdots, f_{m}$ such that

$$
\left|\operatorname{det} \mathcal{K}\left(\zeta_{k}\right)\right| \geq \epsilon_{1} \exp \left(-C_{1} p\left(\zeta_{k}\right)\right)
$$

where $\epsilon_{1}, C_{1}$ are two positive constants independent of $k$.

Proof: For any matrix $A=\left(a_{i, j}\right)$, we define $\|A\|=\sum_{i, j}\left|a_{i, j}\right|$. Then it is easy to verify that

$$
\|A B\| \leq\|A\| \times\|B\|
$$

for any matrices $A$ and $B$ provided that the left hand side of the above inequality makes sense.

Suppose that there exist some constants $\epsilon, C>0$ such that (3.2) holds. Denote $J:=J_{f_{1}, \cdots, f_{m}}$, which is a $m \times n$ matrix-valued function. Then for any $u=\left(u_{1} \cdots u_{n}\right) \in \mathbf{S}^{2 n-1}$, if $J u^{t}=v$, then

$$
\left\|v\left(\zeta_{k}\right)\right\| \geq \epsilon \exp \left(-C p\left(\zeta_{k}\right)\right)
$$

where $u^{t}$ denotes the transpose of $u$. This shows that for each $k$, the kernel of the mapping $\mathcal{J}=J\left(\zeta_{k}\right): \mathbf{C}^{n} \rightarrow \mathbf{C}^{m}$, defined by $\mathcal{J}(u)=J \cdot u^{t}$ for $u=\left(u_{1}, \cdots, u_{n}\right)$, is zero and thus the dimension of the image $\mathcal{J}\left(\mathbf{C}^{n}\right)$ is $n$. Up to an isomorphism, we can identify $\mathcal{J}\left(\mathbf{C}^{n}\right)$ with the space $\mathbf{C}^{n}$. It is then clear that there exists an operator $T: \mathbf{C}^{m} \rightarrow \mathbf{C}^{n}$, given by a $n \times m$ matrix such that $T v^{t}=v^{t}$ for $v \in \mathcal{J}\left(\mathbf{C}^{n}\right)$ and $\|T\| \leq L$ for some constant $L>0$. Let $Q=T \mathcal{J}$. Then it is easy to check that the image $Q\left(\mathbf{C}^{n}\right)$ is the whole space $\mathbf{C}^{n}$. Thus, the matrix $Q$ is invertible. Now set $P=Q^{-1} T$. Then $P \mathcal{J}=(T \mathcal{J})^{-1} T \mathcal{J}=E_{n}$, the $n \times n$ unit matrix. By the well-known Binet-Cauchy theorem (see e.g. [A]), any $r$-rowed determinant of $P \mathcal{J}$ is equal to a sum of terms each of which is a product of an $r$-rowed determinant of $P$ and an $r$-rowed determinant of $\mathcal{J}$. In particular, we have that

$$
\sum_{l=1}^{N}\left(\operatorname{det} P_{l}\right)\left(\operatorname{det} \mathcal{J}_{l}\right)=\operatorname{det} E_{n}=1,
$$


where $N$ is an integer only depending on $n$ and $m$, and $P_{l}$ and $\mathcal{J}_{l}$ are $n \times n$ minors of $P$ and $\mathcal{J}$, respectively. On the other hand, for any $u \in \mathbf{S}^{2 n-1}$, we have that $Q u^{t}=T \mathcal{J} u^{t}=\mathcal{J} u^{t}$ and so that by (3.4),

$$
\left\|Q u^{t}\right\| \geq \epsilon \exp \left(-C p\left(\zeta_{k}\right)\right) .
$$

Notice that $\operatorname{det} Q=\lambda_{1} \cdots \lambda_{n}$, where $\lambda_{j}^{\prime} s$ are eigenvalues of $Q$. Let $u_{\lambda_{j}} \in$ $\mathbf{S}^{2 n-1}$ be the unit eigenvector corresponding to $\lambda_{j}$. Then

$$
Q u_{\lambda_{j}}^{t}=\lambda_{j} u_{\lambda_{j}}
$$

Hence

$$
n\left|\lambda_{j}\right| \geq\left|\lambda_{j}\right||| u_{\lambda_{j}}\|=\| Q u_{\lambda_{j}}^{t} \| \geq \epsilon \exp \left(-C p\left(\zeta_{k}\right)\right)
$$

or

$$
\left|\lambda_{j}\right| \geq \frac{1}{n} \epsilon \exp \left(-C p\left(\zeta_{k}\right)\right)
$$

We then have that

$$
\left|\operatorname{det} Q\left(\zeta_{k}\right)\right| \geq \frac{\epsilon^{n}}{n^{n}} \exp \left(-C n p\left(\zeta_{k}\right)\right) .
$$

It is obvious that

$$
\begin{aligned}
\left\|Q\left(\zeta_{k}\right)\right\| & =\left\|T\left(\zeta_{k}\right) \mathcal{J}\left(\zeta_{k}\right)\right\| \leq\left\|T\left(\zeta_{k}\right)\right\| \times\left\|\mathcal{J}\left(\zeta_{k}\right)\right\| \leq \\
& \leq L\left\|\mathcal{J}\left(\zeta_{k}\right)\right\| \leq L A \exp \left(B p\left(\zeta_{k}\right)\right)
\end{aligned}
$$

for some constants $A, B>0$ since the $f_{1}, \cdots, f_{m}$ are in the space $A_{p}$ and $A_{p}$ is closed under differentiation. Thus, $Q^{*}\left(\zeta_{k}\right) \leq A_{1} \exp \left(B_{1} p(\zeta)\right)$ for some constants $A_{1}, B_{1}>0$, where $Q^{*}$ denotes the adjoint matrix of $Q$. It now follows that

$$
\left\|Q^{-1}\left(\zeta_{k}\right)\right\|=\left\|\frac{Q^{*}\left(\zeta_{k}\right)}{\operatorname{det} Q\left(\zeta_{k}\right)}\right\| \leq A_{2} \exp \left(B_{2} p\left(\zeta_{k}\right)\right)
$$

and thus that

$$
\|P\|=\left\|Q^{-1} T\right\| \leq L\left\|Q^{-1}\right\| \leq L A_{2} \exp \left(B_{2} p\left(\zeta_{k}\right)\right) .
$$

Therefore, each $n \times n$ minor $P_{l}(l=1, \cdots, N)$ of $P$ satisfies that

$$
\left|\operatorname{det} P_{l}\right| \leq A_{3} \exp \left(B_{3} p\left(\zeta_{k}\right)\right) \text {. }
$$

It follows from (3.5) that there exists at least one of $l(l=1, \cdots, N)$ such that

$$
\left|\operatorname{det} \mathcal{J}_{l}\right| \geq \frac{1}{N A_{3}} \exp \left(-B_{3} p\left(\zeta_{k}\right)\right) .
$$


This concludes the proof of the necessity.

Conversely, if (3.3) holds for some $\epsilon_{1}, C_{1}>0$. For any $u \in \mathbf{S}^{2 n-1}$, we let $\mathcal{K} u^{t}=v$. Then $u^{t}=\mathcal{K}^{-1} v$ and thus

$$
\|u\| \leq\left\|\mathcal{K}^{-1}\right\| \times\|v\|, \quad \text { or } \quad\|v\| \geq \frac{\|u\|}{\left\|\mathcal{K}^{-1}\right\|} .
$$

Notice that $\mathcal{K}^{-1}=\frac{\mathcal{K}^{*}}{\operatorname{det} \mathcal{K}}$, where $\mathcal{K}^{*}$ denotes the adjoint matrix of $\mathcal{K}$. Since $A_{p}$ is closed under differentiation, we deduce that

$$
\left\|\mathcal{K}^{*}(\xi)\right\| \leq A_{4} \exp \left(B_{4} p(\xi)\right), \xi \in \mathbf{C}^{n}
$$

and thus that by (3.3),

$$
\left\|\mathcal{K}^{-1}\right\| \leq \frac{A_{4}}{\epsilon_{1}} \exp \left(\left(B_{4}+C_{1}\right) p(\xi)\right)
$$

for some constants $A_{4}, B_{4}>0$. We then obtain that, by (3.6) and taking into account that $\|u\| \geq \frac{1}{\sqrt{n}}$ for any $u \in \mathbf{S}^{2 n-1}$,

$$
\|v\| \geq \epsilon \exp \left(-C p\left(\zeta_{k}\right)\right)
$$

for some constants $\epsilon, C>0$. Hence

$$
\sum_{j=1}^{m}\left|D_{u} f_{j}\left(\zeta_{k}\right)\right|=\left\|\mathcal{J} u^{t}\right\| \geq\left\|\mathcal{K} u^{t}\right\| \geq \epsilon \exp \left(-C p\left(\zeta_{k}\right)\right) .
$$

The proof of the lemma is thus complete.

We are now going to prove Theorem 2.5. In the sequel, we shall use $A$ and $B$ to denote positive constants the actual values of which may vary from one occurrence to the next.

Proof of Theorem 2.5: Sufficiency: For any fixed $k \in \mathbf{N}$, consider the entire function

$f_{j, u}(z):=f_{j}\left(\zeta_{k}+u z\right): \mathbf{C} \rightarrow \mathbf{C}, \quad 1 \leq j \leq m, u=\left(u_{1}, \cdots, u_{n}\right) \in \mathbf{S}^{2 n-1}$.

Then

$$
f_{j, u}^{\prime}(0)=\frac{\partial f_{j}\left(\zeta_{k}\right)}{\partial \xi_{1}} u_{1}+\frac{\partial f_{j}\left(\zeta_{k}\right)}{\partial \xi_{2}} u_{2}+\cdots+\frac{\partial f_{j}\left(\zeta_{k}\right)}{\partial \xi_{n}} u_{n}=D_{u} f_{j}\left(\zeta_{k}\right) .
$$

Therefore, by (2.4),

$$
\sum_{j=1}^{m}\left|f_{j, u}^{\prime}(0)\right| \geq \epsilon \exp \left(-C p\left(\zeta_{k}\right)\right)
$$


It follows then that for any $u \in \mathbf{S}^{2 n-1}$ there exists a $j_{u}$ such that $1 \leq$ $j_{u} \leq m$ and

$$
\left|f_{j_{u}, u}^{\prime}(0)\right| \geq \frac{1}{m} \epsilon \exp \left(-C p\left(\zeta_{k}\right)\right)
$$

Denote

$$
V_{u}=\left\{z \in \mathbf{C}: f_{j_{u}, u}(z)=0\right\}
$$

and

$$
d_{u}=\min \left\{1, \operatorname{dist}\left\{0, V_{u} \backslash\{0\}\right\} .\right.
$$

Since $f_{j_{u}} \in A_{p}$, we have, in $|z| \leq 1$

$$
\left|f_{j_{u}, u}(z)\right|=\left|f_{j_{u}}\left(\zeta_{k}+u z\right)\right| \leq A \exp \left(B p\left(\zeta_{k}+u z\right)\right),
$$

for some constants $A, B>0$ independent of $u$ and $k$. However,

$$
\left|\zeta_{k}+u z-\zeta_{k}\right|=|u z|=|z| \leq 1 .
$$

Thus by the property $(2.2)$ of $p$, we deduce that

$$
p\left(\zeta_{k}+u z\right) \leq C_{1} p\left(\zeta_{k}\right)+C_{2},
$$

with $C_{1}$ and $C_{2}$ being the same as in (2.2). This yields that

$$
\left|f_{j_{u}, u}(z)\right| \leq A \exp \left(B p\left(\zeta_{k}\right)\right), \quad|z| \leq 1
$$

with $A, B>0$ independent of $u, k$. Set

$$
g_{u}(z)=\frac{f_{j_{u}}, u(z)}{z}: \mathbf{C} \rightarrow \mathbf{C} .
$$

Then on $|z|=1$ and so in $|z| \leq 1$ by the Maximum Modulus Theorem,

$$
\left|g_{u}(z)\right| \leq A \exp \left(B p\left(\zeta_{k}\right)\right) .
$$

Therefore, the function

$$
G_{u}(z):=\frac{g_{u}(z)-g_{u}(0)}{3 A \exp \left(B p\left(\zeta_{k}\right)\right)}
$$

satisfies that $G_{u}(0)=0$ and $\left|G_{u}(z)\right|<1$ in $|z|<1$. By the Schwarz Lemma (see e.g. [BG]), $\left|G_{u}(z)\right| \leq|z|$ in $|z|<1$. In particular, letting $a \neq 0$ be any zero of $f_{j_{u}, u}$ in $|z|<1$, then we have, in view of (3.7),

$$
\begin{aligned}
|a| \geq\left|G_{u}(a)\right| & =\left|\frac{g_{u}(0)}{3 A \exp \left(B p\left(\zeta_{k}\right)\right)}\right|= \\
& =\left|\frac{f_{j_{u}, u}^{\prime}(0)}{3 A \exp \left(B p\left(\zeta_{k}\right)\right)}\right| \geq \epsilon_{1} \exp \left(-c_{1} p\left(\zeta_{k}\right)\right)
\end{aligned}
$$


for some positive constants $\epsilon_{1}, c_{1}\left(\epsilon_{1}<1\right)$ which are independent of $u$ and $k$. This implies that

$$
d_{u} \geq \epsilon_{1} \exp \left(-c_{1} p\left(\zeta_{k}\right)\right)
$$

Using the Carathèodory inequality for $g_{u}(z)$ (see e.g. [Le, p. 19]), we have that in $|z| \leq \frac{d_{u}}{2}$,

$$
\log \left|\frac{g_{u}(z)}{g_{u}(0)}\right| \geq-\frac{2 \times \frac{d_{u}}{2}}{d_{u}-\frac{d_{u}}{2}} \log \left(\max _{|z|=d_{u}}\left\{\left|\frac{g_{u}(z)}{g_{u}(0)}\right|\right\}\right)
$$

and so that, by the fact that $g_{u}(0)=f_{j_{u}, u}^{\prime}(0),(3.7)$ and (3.8),

$$
\left|g_{u}(z)\right| \geq \epsilon_{2} \exp \left(-c_{2} p\left(\zeta_{k}\right)\right),
$$

where $\epsilon_{2}, c_{2}$ are positive numbers independent of $k$ and $u$. Let

$$
\hat{d}_{k}=\epsilon_{1} \exp \left(-c_{1} p\left(\zeta_{k}\right)\right),
$$

where $\epsilon_{1}$ and $c_{1}$ are the same as in (3.9). Then for $|z|=\frac{\hat{d}_{k}}{2}$, we have that

$$
\left|f_{j_{u}, u}(z)\right|=\left|z g_{u}(z)\right| \geq \epsilon_{3} \exp \left(-c_{3} p\left(\zeta_{k}\right)\right) .
$$

Thus, we have proved that for any $u \in \mathbf{S}^{2 n-1}$, there exists a $j_{u}$ with $1 \leq j_{u} \leq n$ such that for $|z|=\frac{\hat{d_{k}}}{2}$

$$
\left|f_{j_{u}}\left(\zeta_{k}+u z\right)\right| \geq \epsilon \exp \left(-c p\left(\zeta_{k}\right)\right) .
$$

and so that we always have

$$
\sum_{j=1}^{m}\left|f_{j}\left(\zeta_{k}+u z\right)\right| \geq \epsilon \exp \left(-c p\left(\zeta_{k}\right)\right), \quad u \in \mathbf{S}^{2 n-1},|z|=\frac{\hat{d}_{k}}{2},
$$

for some positive constants $c$ and $\epsilon(\epsilon<1)$ independent of $k$ and $u$.

Now for any $k \in \mathbf{N}$, consider the neighborhood

$$
U_{k}:=\left\{\xi \in \mathbf{C}^{n}:\left|\xi-\zeta_{k}\right| \leq \frac{\hat{d_{k}}}{2}\right\}
$$

of $\zeta_{k}$, where $\hat{d_{k}}$ is defined as above. Note that for any $\xi \in \partial U_{k}$, we have $\xi=\zeta_{k}+\frac{\hat{d_{k}}}{2} u_{\xi-\zeta_{k}}$ for some unit vector $u_{\xi-\zeta_{k}} \in \mathbf{S}^{2 n-1}$. Hence by (3.10), we have that for $\xi \in \partial U_{k}$,

$$
\sum_{j=1}^{m}\left|f_{j}(\xi)\right|=\sum_{j=1}^{m}\left|f_{j}\left(\zeta_{k}+\frac{\hat{d_{k}}}{2} u_{\xi-\zeta_{k}}\right)\right| \geq \epsilon \exp \left(-c p\left(\zeta_{k}\right)\right) .
$$


Let

$$
S\left(f_{1}, f_{2}, \cdots, f_{m} ; \epsilon, c\right)=\left\{\xi \in \mathbf{C}^{n}: \sum_{j=1}^{m}\left|f_{j}(\xi)\right|<\epsilon \exp (-c p(\xi))\right\} .
$$

The component of $S\left(f_{1}, f_{2}, \cdots, f_{m} ; \epsilon, c\right)$ containing $\zeta_{k}$ is denoted by $\hat{V}_{k}$. Then obviously, $\hat{V}_{k} \subset U_{k}$ by (3.11). We claim that $U_{k}$ does not contain any $\zeta_{j}$ if $j \neq k$. In fact, letting $d_{j}^{\prime}=\left|\zeta_{j}-\zeta_{k}\right|$, we know that $\zeta_{j}=$ $\zeta_{k}+d_{j}^{\prime} u_{\zeta_{j}-\zeta_{k}}$ for some unit vector $u_{\zeta_{j}-\zeta_{k}} \in \mathbf{S}^{2 n-1}$. By the definition of $d_{u}$ and (3.9), we deduce that

$$
d_{j}^{\prime} \geq d_{u_{\zeta_{j}-\zeta_{k}}} \geq \epsilon_{1} \exp \left(-c_{1} p\left(\zeta_{k}\right)\right)=\hat{d}_{k} .
$$

This implies that $\zeta_{j} \notin U_{k}$.

Thus for any sequence $\left\{a_{k}\right\} \in A_{p}(V)$, we can define an analytic function $F(\xi): S\left(f_{1}, \cdots, f_{m} ; \epsilon, c\right) \mapsto \mathbf{C}$ by:

$$
F(z)= \begin{cases}a_{k}, & \text { if } z \in \hat{V}_{k} \\ 0, & \text { if } z \in S\left(f_{1}, \cdots, f_{m} ; \epsilon, c\right) \backslash \cup_{k \in \mathbf{N}} \hat{V}_{k} .\end{cases}
$$

Then the sufficiency follows from the following semi-local to global extension theorem ([BT2]): suppose that for some $\epsilon, c>0 \lambda$ is a function analytic on $S\left(f_{1}, \cdots, f_{m} ; \epsilon, c\right)$ such that

$$
|\lambda(\xi)| \leq A_{1} \exp \left(B_{1} p(\xi)\right)
$$

for all $\xi \in S\left(f_{1}, \cdots, f_{m} ; \epsilon, c\right)$. Then there exists an entire function $f \in$ $A_{p}$, constants $\epsilon_{1}, c_{1}, A, B>0$ such that for $\xi \in S\left(f_{1}, \cdots, f_{m} ; \epsilon, c\right)$,

$$
f(\xi)-\lambda(\xi)=\left(g_{1} f_{1}+\cdots+g_{m} f_{m}\right)(\xi)
$$

and $\left|g_{j}(\xi)\right| \leq A \exp (B p(\xi))$ for all $\xi \in S\left(f_{1}, \cdots, f_{m} ; \epsilon_{1}, c_{1}\right)$. In particular, $f(\xi)=\lambda(\xi)$ on $Z\left(f_{1}, \cdots, f_{m}\right)$. Applying this result with $\lambda(\xi)=F(\xi)$, we then obtain an entire function $f \in A_{p}\left(\mathbf{C}^{n}\right)$ such that $f\left(\zeta_{k}\right)=F\left(\zeta_{k}\right)=a_{k}$. This completes the proof of sufficiency.

Necessity: For any $M>0$, we set

$$
E=\left\{a=\left\{a_{k}\right\}_{k \in \mathbf{N}}:\left|a_{k}\right| \exp \left(-M p\left(\zeta_{k}\right)\right) \leq 1, k \in \mathbf{N}\right\} .
$$

Then the space $E$ is complete under the metric induced by the norm

$$
\|a\|_{E}:=\sup \left\{\left|a_{k}\right| \exp \left(-M p\left(\zeta_{k}\right)\right): k \in \mathbf{N}\right\} .
$$


For $l \in \mathbf{N}$, let

$$
E_{l}=\left\{f \in \mathbf{A}_{p}:|f(\xi)| \leq l \exp (l p(\xi))\right\} .
$$

By the hypothesis that $\left\{\zeta_{k}\right\}$ is an interpolating variety for $A_{p}\left(\mathbf{C}^{n}\right)$, we see that $E=\cup_{l=1}^{\infty} \hat{E}_{l}$, where $\hat{E}_{l}:=\left\{\left\{f\left(\zeta_{k}\right)\right\} \in E: f \in E_{l}\right\}$.

We next prove that each $\hat{E}_{l}$ is closed subset of $E$. In fact, suppose that $f_{j}$ is a sequence of functions in $E_{l}$ satisfying that for each $j,\left\{f_{j}\left(\zeta_{k}\right)\right\} \in E$ and

$$
\left\{f_{j}\left(\zeta_{k}\right)\right\} \rightarrow a \in E, \quad \text { as } \quad j \rightarrow \infty .
$$

Since $\left|f_{j}(\xi)\right| \leq l \exp (l(p(\xi))$ for any $j \in \mathbf{N}$ and the weight $p$ has the property (2.2), $\left\{f_{j}\right\}$ is locally bounded. By the $\mathbf{C}^{n}$ version of Montel's theorem (see e.g. [G, p. 54]), $\left\{f_{j}\right\}$ is a normal family. By passing to subsequence, we can assume, without loss of generality, that $f_{j} \rightarrow f$ normally. Now, it follows from the Weierstrass theorem that $f$ is an entire function on $\mathbf{C}^{n}$. It is clear that $f \in E_{l}$ and $\left\{f\left(\zeta_{k}\right)\right\}=a$ by the uniqueness of limit. Thus $a \in \hat{E}_{l}$. That is, $\hat{E}_{l}$ is closed. We conclude by the the Baire-category theorem (see e.g. $[\mathbf{L} \mathbf{a}]$ ) that some $\hat{E}_{l}$ has non-empty interior. It is no loss of generality to assume that

$$
\hat{E}_{l} \supset\left\{a \in E:\|a\|_{E}<\epsilon\right\}
$$

for some $\epsilon>0$, from which we readily obtain a sequence $\left\{h_{k}\right\}$ with $h_{k} \in E_{l}$ such that

$$
h_{k}\left(\zeta_{k}\right)=\epsilon \exp \left(M p\left(\zeta_{k}\right)\right) \text { and } h_{k}\left(\zeta_{j}\right)=0, j \neq k .
$$

Recall that $\mathbf{S}^{2 n-1}=\left\{u=\left(u_{1}, \cdots, u_{n}\right) \in \mathbf{C}^{n}:|u|^{2}=\left(\left|u_{1}\right|^{2}+\cdots+\left|u_{n}\right|^{2}\right)^{\frac{1}{2}}=\right.$ $1\}$. We can cover $\mathbf{S}^{2 n-1}$ by its $n$ subsets

$$
\Delta_{j}:=\left\{u=\left(u_{1}, \cdots, u_{n}\right) \in \mathbf{S}^{2 n-1}:\left|u_{j}\right| \geq \frac{1}{\sqrt{n}}\right\}
$$

$(1 \leq j \leq n)$. That is, $\mathbf{S}^{2 n-1}=\cup_{j=1}^{n} \Delta_{j}$. For each fixed $j(1 \leq j \leq n)$, we define for $\xi \in \mathbf{C}^{n}$,

$$
f_{j}(\xi)=\sum_{k=1}^{\infty}\left(\xi_{j}-\zeta_{k, j}\right) h_{k}^{2}(\xi) / \exp \left(2 M p\left(\zeta_{k}\right)\right), \quad 1 \leq j \leq n,
$$

where $\xi=\left(\xi_{1}, \cdots, \xi_{n}\right)$ and $\zeta_{k}=\left(\zeta_{k, 1}, \cdots, \zeta_{k, n}\right)$. We shall prove that for each $j$, the above series converges to an entire function $f_{j}$ in $A_{p}\left(\mathbf{C}^{n}\right)$. Assuming this for the moment, we see that

$$
V \subset Z\left(f_{1}, f_{2}, \cdots, f_{n}\right)
$$


and $f_{j}$ can be expanded into the following power series at each $\zeta_{k}$,

$$
\begin{aligned}
& f_{j}(\xi)=\epsilon^{2}\left(\xi_{j}-\zeta_{k, j}\right)+ \\
& +\sum_{i_{1}+\cdots+i_{n}=1}^{\infty} C_{i_{1}, \cdots, i_{n}}\left(\xi_{1}-\zeta_{k, 1}\right)^{i_{1}} \cdots\left(\xi_{j}-\zeta_{k, j}\right)^{i_{j}+1} \cdots\left(\xi_{n}-\zeta_{k, n}\right)^{i_{n}} .
\end{aligned}
$$

From this, it follows that

$$
\nabla f_{j}\left(\zeta_{k}\right):=\left(\frac{\partial f_{j}}{\partial \xi_{1}}\left(\zeta_{k}\right), \cdots, \frac{\partial f_{j}}{\partial \xi_{n}}\left(\zeta_{k}\right)\right)=\left(0, \cdots, 0, \epsilon^{2}, 0, \cdots, 0\right)
$$

with the $j$-th entry being $\epsilon^{2}$. On the other hand, for any $u \in \mathbf{S}^{2 n-1}$, there exists a $j(1 \leq j \leq n)$ such that $u \in \Delta_{j}$ and so that $\left|u_{j}\right| \geq \frac{1}{\sqrt{n}}$. Hence by (3.15)

$$
\begin{aligned}
\sum_{i=1}^{n}\left|D_{u} f_{i}\left(\zeta_{k}\right)\right| & \geq\left|D_{u} f_{j}\left(\zeta_{k}\right)\right|= \\
& =\left|u_{1} \frac{\partial f_{j}}{\partial \xi_{1}}\left(\zeta_{k}\right)+\cdots+u_{j} \frac{\partial f_{j}}{\partial \xi_{j}}\left(\zeta_{k}\right)+\cdots+u_{n} \frac{\partial f_{j}}{\partial \xi_{n}}\left(\zeta_{k}\right)\right|= \\
& =\left|u_{j} \frac{\partial f_{j}}{\partial \xi_{j}}\left(\zeta_{k}\right)\right|=\epsilon^{2}\left|u_{j}\right| \geq \epsilon^{2} \frac{1}{\sqrt{n}} .
\end{aligned}
$$

Thus, the necessity will follow from (3.14) and (3.16) once we show that each $f_{j} \in A_{p}$ for $1 \leq j \leq n$ (if $m>n$, we can easily add $m-n$ functions $f_{n+1}, \cdots f_{m}$ such that (2.3) and (2.4) hold). In fact, since $h_{k} \in E_{l}$ for any $k \in \mathbf{N}$, we have for any $\xi \in \mathbf{C}^{n}$

$$
\left|h_{k}(\xi)\right| \leq l \exp (l p(\xi)) .
$$

Also,

$$
\begin{aligned}
\left|\xi_{j}-\zeta_{k, j}\right| & \leq\left|\xi-\zeta_{k}\right| \leq|\xi|+\left|\zeta_{k}\right| \leq \\
& \leq A \exp (B p(\xi))+A \exp \left(B p\left(\zeta_{k}\right)\right)
\end{aligned}
$$

for some positive constants $A, B$ by the property (2.1) of the weight $p$. Thus, for any $k \in \mathbf{N}$

$$
\left|\left(\xi_{j}-\zeta_{k, j}\right) h_{k}^{2}(\xi) / \exp \left(2 M p\left(\zeta_{k}\right)\right)\right| \leq A \exp (B p(\xi)) \exp \left((C-2 M) p\left(\zeta_{k}\right)\right),
$$

for some constants $A, B, C>0$ with $C$ being independent of $M$. We claim that

$$
\sum_{k=1}^{\infty} \exp \left((C-2 M) p\left(\zeta_{k}\right)\right)<\infty
$$


provided that $M$ is large. To this end, let

$$
\delta_{k}=\inf _{j \neq k}\left\{\left|\zeta_{j}-\zeta_{k}\right|\right\}, \quad \eta_{k}=\min \left\{1, \delta_{k}\right\}
$$

and

$$
\mathcal{B}_{k}=\mathcal{B}\left(\zeta_{k}, \eta_{k}\right):=\left\{\xi \in \mathbf{C}^{n}:\left|\xi-\zeta_{k}\right| \leq \eta_{k}\right\} .
$$

By repeating the proof of (3.12) for $M=1$, one can obtain another sequence $\left\{g_{k}\right\}$ of entire functions satisfying that

$$
g_{k}\left(\zeta_{k}\right)=\epsilon_{1}, \quad g_{k}\left(\zeta_{j}\right)=0, j \neq k,
$$

where $\epsilon_{1}$ is a positive number, and for $\xi \in \mathbf{C}^{n}, k \in \mathbf{N}$

$$
\left|g_{k}(\xi)\right| \leq A \exp (B p(\xi))
$$

for some positive numbers $A$ and $B$. Denote $G_{k}(\xi)=g_{k}(\xi)-g_{k}\left(\zeta_{k}\right)$. Then $G_{k}\left(\zeta_{k}\right)=0$ and for $\left|\xi-\zeta_{k}\right| \leq 1$

$$
\left|G_{k}(\xi)\right| \leq\left|g_{k}(\xi)\right|+\left|g_{k}\left(\zeta_{k}\right)\right| \leq A_{1} \exp \left(B_{1} p\left(\zeta_{k}\right)\right)
$$

for some constants $A_{1}, B_{1}>0$, in view of (3.19) and the property (2.2) of $p$. Recall the following $\mathbf{C}^{n}$ version of Schwarz's Lemma (c.f. [G, p. 7]): If $F$ is holomorphic in an open neighborhood of a closed ball $\overline{\mathcal{B}}(\zeta, r)$ and $|F(\xi)| \leq \alpha$ for all $\xi \in \overline{\mathcal{B}}(\zeta, r)$, and if $\frac{\partial^{|I|} F}{\partial \xi^{T}}(\xi)=0$ whenever $|I|<m$ for some $m \in \mathbf{N}$, where $I=\left(i_{1}, i_{2}, \cdots, i_{n}\right)$ is a multi-index, $|I|=i_{1}+\cdots+i_{n}$, then

$$
|F(\xi)| \leq \alpha r^{-m}|\xi-\zeta|^{m}, \quad \xi \in \overline{\mathcal{B}}(\zeta, r) .
$$

Applying this result to the function $G_{k}(\xi)$ with $m=1$, we have that for $\xi \in \overline{\mathcal{B}}\left(\zeta_{k}, 1\right)$,

$$
\left.\mid G_{(} \xi\right)\left|\leq A_{1} \exp \left(B_{1} p\left(\zeta_{k}\right)\right)\right| \xi-\zeta_{k} \mid
$$

for some positive constants $A_{1}, B_{1}$. In particular, if $\zeta_{j} \in \overline{\mathcal{B}}\left(\zeta_{k}, 1\right), j \neq k$, then we have that, in view of (3.18),

$$
\epsilon_{1}=\left|g_{k}\left(\zeta_{k}\right)\right|=\left|G_{k}\left(\zeta_{j}\right)\right| \leq A_{1} \exp \left(B_{1} p\left(\zeta_{k}\right)\right)\left|\zeta_{j}-\zeta_{k}\right|
$$

or

$$
\left|\zeta_{j}-\zeta_{k}\right| \geq \epsilon_{1} A_{1}^{-1} \exp \left(-B_{1}\left(\zeta_{k}\right)\right)
$$

This shows that

$$
\delta_{k} \geq \epsilon_{1} A_{1}^{-1} \exp \left(-B_{1} p\left(\zeta_{k}\right)\right)
$$


It now follows from Lemma 3.1 that

$$
\sum_{k=1}^{\infty} \exp \left((C-2 M) p\left(\zeta_{k}\right)\right):=D<\infty
$$

provided that $M$ is large. Combining this result with (3.17), we know that the series (3.13) is uniformly convergent in compact sets of $\mathbf{C}^{n}$ and so $f_{j}$ is an entire function on $\mathbf{C}^{n}$. Furthermore,

$$
\left|f_{j}(\xi)\right| \leq A \exp (B p(\xi)) \times D
$$

that is, $f_{j} \in A_{p}\left(\mathbf{C}^{n}\right)$.

The proof of Theorem 2.5 is thus complete.

Proof of Corollary 2.6: The corollary follows directly from Theorem 2.5 and Lemma 3.2.

Proof of Corollary 2.7: The corollary is obtained from Corollary 2.6 by taking $m=n$.

\section{References}

[A] A. C. Aitken, "Determinants and matrices," Interscience Publishers, Inc., New York, 1962.

[BG] C. A. Berenstein And G. GAY, "Complex variables, an introduction," Springer-Verlag, New York, 1991.

[BL1] C. A. Berenstein AND B. Q. LI, Interpolating varieties for spaces of meromorphic functions, to appear in J. Geometric Analysis.

[BL2] C. A. Berenstein AND B. Q. LI, Interpolation problems with growth conditions for entire functions in one and several complex variables, preprint, 1993.

[BLV] C. A. Berenstein, B. Q. Li and A. Vidras, Geometric characterization of interpolating varieties for the $(F N)$-space $A_{p}^{0}$ of entire functions, to appear in Canadian J. Math..

[BT1] C. A. Berenstein AND B. A. TAYLOR, A new look at interpolating theory for entire functions of one variable, Advances in Math. 33 (1979), 109-143.

[BT2] C. A. BEREnStein AND B. A. TAYLOR, Interpolation problems in $\mathbf{C}^{n}$ with applications to harmonic analysis, J. Analyse Math. $\mathbf{3 8}$ (1981), 188-254. 
[BT3] C. A. Berenstein and B. A. TAYlor, "On the geometry of interpolating varieties," Sem. Lelong-Skoda (1980/1981), Lecture Notes in Math. 919, Springer-Verlag, 1983, pp. 1-25.

[E] L. EHRENPREIS, "Fourier analysis in several variables," Interscience, New York, 1970.

[EM] L. EHRENPREIS AND P. MALLIAVIN, Invertible operators and interpolation in $A U$ spaces, J. Math. Pure. Appl. 53 (1974), 165-182.

[G] R. Gunning, "Introduction to holomorphic functions of several variables," Vol.I, Wadsworth, Inc., Califonia, 1990.

[Ho] L. HöRMANDER, Generators for some rings of analytic functions, Bull. Amer. Math. Soc. 73 (1967), 943-949.

[La] S. LANG, "Real Analysis," Addison-Wesley, 1983.

[L] A. F. LEONT'EV, Representation of functions by generalized Dirichlet series, Russian Math. Surveys 24 (1969), 101-178.

[Le] B. J. Levin, "Distribution of zeros of entire functions," Amer. Math. Soc., Providence, R.I., 1964.

\footnotetext{
Department of Mathematics and Institute for Systems Research

University of Maryland

College Park, MD, 20742

U.S.A.
}

Rebut el 2 de Setembre de 1993 